\title{
Rarefied aerodynamic characteristics of aero-space-planes: a comparative study of two gas-surface interaction models
}

\author{
Gennaro Zuppardi a,*, Luigi Morsa ${ }^{a}$, Raffaele Savino ${ }^{a}$, Martin Sippel ${ }^{\mathrm{b}}$, \\ Tobias Schwanekamp ${ }^{\text {b }}$ \\ a Department of Industrial Engineering, University of Naples "Federico II", 80125 Naples, Italy \\ b Space Launcher System Analysis (SART) DLR, 28359 Bremen, Germany
}

\section{A R T I C L E I N F O}

\section{Article history:}

Received 26 November 2014

Received in revised form

1 April 2015

Accepted 10 April 2015

Available online 18 April 2015

\section{Keywords:}

Gas-surface interaction models

Direct simulation Monte Carlo method

Aerodynamic coefficients of an

aero-space-plane

\begin{abstract}
A B S T R A C T
Accurate computations of aerodynamic forces are very important for the design of aero-space-planes. In rarefied flow, the computation of momentum and energy transfer between the flow and the vehicle's surface occurs in two steps: incidence and re-emission of gas molecules. While incidence is well understood, the re-emission process is still today not yet completely clear, thus many models have been developed. In the present paper, the effects of the re-emission models by Maxwell and by Cercignani-Lampis-Lord have been compared by means of direct simulation Monte Carlo (DSMC) codes. Two different study cases have been considered: a complete aero-space-plane and a wing profile. Computer simulations have been carried out using two DSMC codes to investigate hypersonic flows at an altitude of $120 \mathrm{~km}$ where for both the wing section and the vehicle the flow is in transitional regime. The results pointed out that the influence of the interaction models, considering specular, semi-specular and full diffusive re-emission is pretty strong, while the Cercignani-Lampis-Lord and the Maxwell models are almost equivalent.
\end{abstract}

(c) 2015 Elsevier Masson SAS. All rights reserved.

\section{Introduction}

The computation of aerodynamic characteristics of aero-spaceplanes in high altitude flight, or in rarefied flows, relies on the Direct Simulation Monte Carlo (DSMC) method [1-3]. A correct evaluation of the aerodynamic force and moments of a spacecraft along its ascent and/or re-entry path provides useful information for the proper design of the propulsion and attitude control systems, for instance to ensure trim, stability and maneuverability. In rarefied flow, accurate computations rely on a proper choice of the gas-surface interaction model. This influences the computation of momentum and energy exchanged with the surface and therefore the aerodynamic forces, moments and heat flux.

It is well known that, in rarefied flow, the gas-surface interaction is considered as composed of two different mechanisms: incidence and re-emission. While incidence is well understood and the related computation has been proved to be accurate, the reemission phase is still today not yet completely understood; thus many models (Maxwell [1-3], Cercignani-Lampis [1-3], Schamberg [4,5], Nocilla [6]) have been proposed, trying to describe the

\footnotetext{
* Corresponding author.

E-mail address: zuppardi@unina.it (G. Zuppardi).
}

molecule remission from the surface of the body. Every model is based on simplifying assumptions and relies on "engineering" parameters (or accommodation coefficients) providing against the ignorance about the physical mechanism of the re-emission process. Lord [1,2,7] provided a DSMC implementation of the Cercignani-Lampis model, thus the model is labeled as CLL.

Most of the DSMC computations currently are based on the assumption of full diffusive interaction. According to Bird [1,2], this assumption should be reviewed whenever a smooth surface has been exposed for long time to ultra-high vacuum, a surface is heated, the weight of the gas molecule is much smaller than the weight of the surface material, the translational energy of the impinging molecules is larger than several electron-volts. Knechtel and Pitts [8] verified experimentally that the accommodation coefficients of the normal and tangential momentum components depend on a number of factors: gas composition, impact energy of the impinging molecules, surface temperature, surface material, roughness of the surface, angle of incidence. These measurements are particularly interesting because pretty close to space applications.

The present paper is the follow-on of a former article by Zuppardi [9] where the Maxwell and the Schamberg models were compared with DSMC computations carried out only at the impact point on an elementary surface changing the incidence angle. In 
the present work, the Maxwell and CLL models are compared considering:

1. the impact point on an elementary surface, for a basic, direct comparison of the re-emitted quantities, including the visualization of the molecule re-mission lobes.

2. a wing section of SpaceLiner, an advanced visionary concept of suborbital, hypersonic, winged passenger transport, currently under investigation at DLR, Space Launcher System Analysis [10-12]. In this case computations are aimed at the evaluation of the influence of the two interaction models on global and local aerodynamic coefficients.

3. the complete SpaceLiner vehicle in clean and flapped configurations, to assess the effect of the interaction model on the aerodynamic coefficients, on the lift-to-drag ratio and on the pitching moment around the center of gravity of the vehicle. The latter is particularly important to assess the vehicle's longitudinal stability and maneuverability.

Two dimensional and three dimensional computer tests were carried out by the DS2V-64 bits [13] and DS3V [14] codes, including the Maxwell and CLL models with specular, semi-specular and full diffusive molecule-surface interactions. The models assume constant accommodation coefficients on the whole surface, i.e. independent from the incidence angle. Unfortunately, the lack of experimental data in real conditions does not make possible a proper evaluation of the models; thus the scope of the paper is only to verify and quantify the differences in results obtained with the two interaction models and with different accommodation coefficients.

The influence of the gas-surface interaction on the aerodynamic coefficients has been already considered for simple geometrical shapes, as per satellites, with the many purposes:

1. to investigate the effect of the interaction model on the aerodynamic drag,

2. to study satellite dynamics [15],

3. to evaluate atmospheric density $[16,17]$.

A direct, quantitative comparison of the results from a DSMC code implementing the Maxwell and CLL models has been already carried out by Padilla and Boyd [18], who investigated aerodynamic forces and heat transfer on a flat plate in a space environment, for a range of angle of attack and with several accommodation coefficients. To the authors knowledge, a systematic study on complex configurations such as winged spacecrafts, has not been considered before.

\section{Maxwell and Cercignani-Lampis models}

As discussed before, the gas-surface interaction includes incidence and re-emission of the molecule upon and from the surface. Molecules impinging upon the surface are considered in equilibrium at temperature $T_{i}$ and molecules re-emitted from the surface are considered in equilibrium at temperature $T_{r}$ that can be different from the surface temperature $T_{w}$. An evaluation of the level at which $T_{r}$ is adjusted toward $T_{w}$ is provided by the thermal accommodation coefficient $\left(\alpha_{E}\right)$ :

$\alpha_{E}=\frac{E_{i}-E_{r}}{E_{i}-E_{w}}$

$E$ is the molecule transitional energy, subscripts $i, r$ and $w$ are for incident, re-emitted and re-emitted at wall temperature. According to Bird [1,2], the energy accommodation coefficient can be approximated by the fraction of molecules reflected diffusely. The

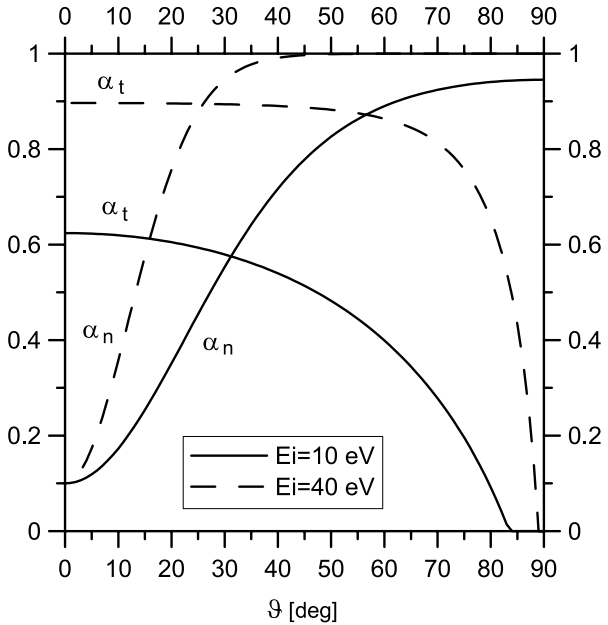

Fig. 1. Profiles of normal $\left(\alpha_{n}\right)$ and tangential $\left(\alpha_{t}\right)$ momentum accommodation coefficients as functions of the local angle of incidence (Ref. [8])

accommodation coefficients are defined also in terms of the normal $\left(\alpha_{n}\right)$ and tangential $\left(\alpha_{t}\right)$ momentum components:

$\alpha_{n}=\frac{p_{i}-p_{r}}{p_{i}-p_{w}}$

$\alpha_{t}=\frac{\tau_{i}-\tau_{r}}{\tau_{i}}$

where $p$ is the pressure and $\tau$ is the shear stress.

All accommodation coefficients range from 0 for no accommodation (specular reflection) to 1 for complete accommodation (full diffusive re-emission). Knechtel and Pitts [8] obtained the following formulae, evaluating $\alpha_{n}$ and $\alpha_{t}$ in the range of the angle of incidence $0 \leq \vartheta \leq 90 \mathrm{deg}$ :

$\alpha_{n}=1.00-0.9 \exp \left(-0.280 E_{c} \cos ^{2} \varphi\right)$

$\alpha_{t}=0.90-1.20 \exp \left(-0.147 E_{c} \sin ^{3 / 4} \varphi\right)$

where $\varphi$ is the complementary of the incidence angle $\vartheta(\varphi=$ $\pi / 2-\vartheta)$. Eqs. (2) are least square curves fitting experimental results obtained for satellites or spacecrafts close to Earth re-entry. For completeness, Fig. 1 shows the profiles of $\alpha_{n}$ and $\alpha_{t}$ as functions of $\vartheta$ and clearly verifies the strong dependence of the momentum accommodation coefficients on the angle of incidence and on the impact energy.

The DSMC implementation of both the Maxwell and the Cercignani-Lampis-Lord model starts from the computation, for each collision, of the incident velocity $V_{i}\left(u_{i}, v_{i}, w_{i}\right)$ of each molecule impinging upon the surface. According to Bird [1,2], the incident velocity is composed of the free stream velocity $V_{\infty}\left(V_{\infty x}, V_{\infty y}, V_{\infty z}\right)$ and the molecular thermal velocity $C(U, V, W)$, set in turn at random from the most probable molecular velocity $(c)$ at temperature $T_{\infty}$ :

$c\left(T_{\infty}\right)=\sqrt{2 \frac{k}{m} T_{\infty}}$

where $k$ is the Boltzmann constant and $m$ is the mass of the molecule:

$U, V, W=\sin (2 \pi R) c\left(T_{\infty}\right) \sqrt{-\ln R}$

$R$ is a random number $(0 \leq R \leq 1)$, different in each formula:

$u_{i}=V_{\infty x}+U$

$v_{i}=V_{\infty y}+V$

$w_{i}=V_{\infty z}+W$. 
The component $u_{i}$ is normal to the surface, the components $v_{i}$ and $w_{i}$ are tangent to the surface.

According to Maxwell, the reflection is governed by the "classical" specular and/or diffusive models. The specular model assumes that the normal component of the reflected velocity is reversed: $u_{r}=-u_{i}$, and the tangential components do not change: $v_{r}=v_{i}$, $w_{r}=w_{i}$. In the DSMC implementations, the diffusive model is considered always full diffusive at the surface temperature. Components of the reflected velocity are computed at random from the most probable molecular velocity in equilibrium at the wall temperature $\left(c\left(T_{w}\right)\right.$, Eq. (3)):

$U_{r}=c\left(T_{w}\right) \sqrt{-\ln R}$

$V_{r}=c\left(T_{w}\right) \sqrt{-\ln R}^{*} \sin (2 \pi R)$

$W_{r}=c\left(T_{w}\right) \sqrt{-\ln R}^{*} \cos (2 \pi R)$.

The normal and tangential components of the stress are linked to the incident and reflected momentum; the resultant pressure and shear stress read:

$p=(1+f) p_{i}+(1-f) p_{u}$

$\tau=(1-f) \tau_{i}$

where $f$ is the fraction of particles re-emitted specularly; therefore a specular re-emission is defined by $f=1$ and a full diffusive remission by $f=0$.

Besides the Maxwell specular and full diffusive models, the Cercignani-Lampis model seems to be the most successful for DSMC application. The model satisfies the reciprocity condition, i.e. the principle of detailed balance; this means that there is a relationship between the probability of a gas-surface interaction with a set of incident and reflected velocities and the probability of the inverse interaction. This model is based on the accommodation coefficient for the kinetic energy $\left(\alpha_{E n}\right)$ associated with the normal component of velocity, and the tangential momentum accommodation, respectively. The model assumes that there is no coupling between the normal and tangential components of the velocity during the reflection process. It provides the probabilities that an incident molecule with normal velocity component $U_{i}$ is reflected with normal velocity component $U_{r}$. The tangential component $V_{i}$ (or $W_{i}$ ) is reflected with tangential velocity $V_{r}\left(\right.$ or $\left.W_{r}\right)$ :

$$
\begin{aligned}
P\left(U_{r}, U_{i}\right)= & \left(\frac{2 U_{r}}{\alpha_{E n}}\right) I_{0}\left\{\frac{2\left(1-\alpha_{E n}\right)^{1 / 2} U_{i} U_{r}}{\alpha_{E n}}\right\} \\
& \times \exp \left\{\frac{U_{r}^{2}+\left(1-\alpha_{E n}\right) U_{i}^{2}}{\alpha_{E n}}\right\}
\end{aligned}
$$

$P\left(V_{r}, V_{i}\right)=\left(\pi \alpha_{t}\right)^{-1 / 2} \exp \left[-\frac{\left\{V_{r}-\left(1-\alpha_{t}\right)^{1 / 2} V_{i}\right\}^{2}}{\alpha_{t}}\right]$

where the components of velocity are made non-dimensional by $c\left(T_{w}\right)$ and $I_{0}$ is a Bessel function of zeroth order. Eq. (8b) holds also for $P\left(W_{r}, W_{i}\right)$. Considering that the energy accommodation coefficient is related only to the kinetic energy, in the present computations, $\alpha_{E n}$ is approximated by $\alpha_{n}: \alpha_{E n} \cong \alpha_{n}$. As the Cercignani-Lampis model relies on the accommodation coefficients $\alpha_{n}$ and $\alpha_{t}$, it overcomes the limitation of the unreal Maxwell specular and full diffusive models.

The DSMC implementation by Lord consists in the following equations computing the components of the reflected velocity:

$U_{r}=\left(r^{2}+(1-\alpha) U_{i}^{2}+2 r(1-\alpha)^{1 / 2} U_{i} \cos \theta\right)^{1 / 2}$

$V_{r}=(1-\alpha)^{1 / 2} V_{i}+r \cos \theta$

$W_{r}=r \sin \theta$ where $\theta$ and $r$ are random quantities:

$\theta=2 \pi R$

$r=\sqrt{-\alpha \ln (R)}$

and $\alpha=\alpha_{n}$ for the normal component $\left(U_{r}\right), \alpha=\alpha_{t}\left(2-\alpha_{t}\right)$ for the tangential components $\left(V_{r}\right.$ and $\left.W_{r}\right)$.

The CLL model verified [19] to provide a realistic boundary condition with incomplete accommodation. In addition it reduces to the specular and to the diffusive model when both $\alpha_{n}$ and $\alpha_{t}$ are zero or unity, respectively. The resultant pressure and shear stress read:

$p=p_{i}+p_{r}$

$\tau=\tau_{i}-\tau_{r}$.

\section{Direct simulation Monte Carlo codes}

It is well known that the DSMC method [1-3] is currently the only tool for the solution of rarefied flow fields from slip flow to free molecular regimes. The Navier-Stokes equations fail in low density regimes due to the failure of the "classical" laws by Newton, Fourier and Fick, computing the transport parameters.

DSMC considers the gas as made up of particles (molecules and atoms). It is based on the kinetic theory of gases and computes the evolution of millions of simulated particles, each one representing a large number (say $10^{15}$ ) of real particles in the physical space. Intermolecular and molecule-surface collisions are taken into account. The computational domain is divided into cells. The cells are used for selecting the colliding molecules and for sampling the macroscopic fluid-dynamic quantities. The most important advantage is that the method does not suffer from numerical instabilities and does not rely on similarity parameters, like the Mach number and the Reynolds number. On the other hand, the method is inherently unsteady; a steady solution is achieved after a sufficiently long simulation time.

The present analysis relies on three DSMC codes:

(1) a code, written specifically for the purpose of this paper, including Eqs. (3)-(10) for the computation of the pressure and tangential stress at the molecule impact point on an elementary surface, changing the incidence angle. The model does not consider intermolecular collisions; the code is provided of a graphical routine for the visualization of the re-emission lobes in the meridian and azimuth planes. The output pressure and shear stress are averaged on the input number of collisions.

(2) DS2V-64 bits [13] to compute, besides the 2-D global aerodynamic coefficients, also the pressure, shear stress along the surface of a wing section of the SpaceLiner vehicle.

(3) DS3V [14] to compute the global aerodynamic coefficients of SpaceLiner in clean and flapped configuration.

Both DS2V and DS3V consider air as made up of five neutral reacting species $\left(\mathrm{O}_{2}, \mathrm{~N}_{2}, \mathrm{O}, \mathrm{N}\right.$ and $\left.\mathrm{NO}\right)$ and rely on the built-in GuptaYos-Thompson [20] chemical model, consisting of 23 reactions. Both codes are "sophisticated". As widely reported in literature [21-24], these codes implement computing procedures providing efficiency and accuracy higher than those from a "basic" DSMC code. A sophisticated code, in fact, is based on two sets of cells (collision and sampling cells) with the related cell adaptation and implements methods promoting nearest neighbor collisions. This type of code generates automatically computational parameters such as numbers of cells and of simulated particles by the input numbers of megabytes and of free stream number density. It uses a radial weighting factor in solving axial-symmetric flow fields and provides optimal time step. Finally, the same collision pair cannot have sequential collisions. 


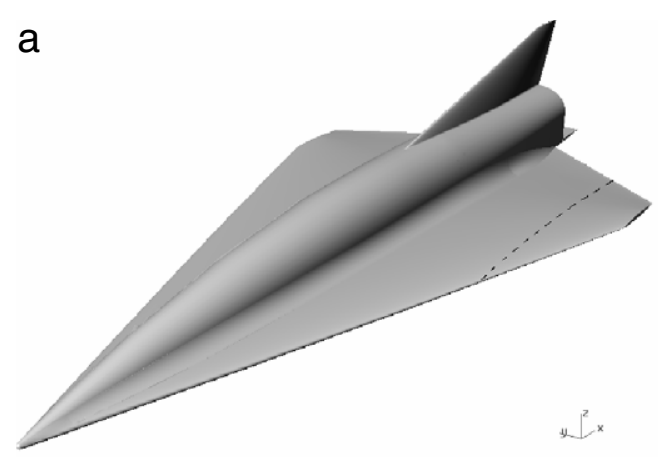

b

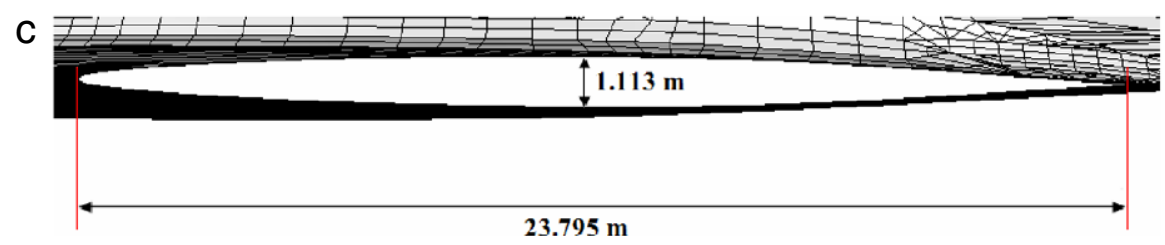

Fig. 2. (a) Rendering of SpaceLiner-7.1, (b) unstructured body grid with deflected flaps used in DS3V simulations ( $\delta=-35$ deg), (c) wing section at $y=11.5 \mathrm{~m}$ from the symmetry plane.

Besides being "sophisticated", DS2V and DS3V are also "advanced", allowing the user to evaluate the quality of a simulation. The user can verify, by the online visualization of the ratio between the molecule mean collision separation (mcs) and the mean free path ( $\lambda$ or m.f.p.) in each computational cell, that the number of simulated particles and the number of collision cells are adequate. In addition, the codes allow the user to change (or to increase), during a run, the number of simulated particles. The ratio mcs $/ \lambda$ has to be less than unity everywhere in the computational domain. Bird $[13,14]$ suggests 0.2 as a limit value for an optimal quality of the run. In addition, the codes give the user information about the stabilization of the runs by means of the profile of the number of simulated particles as a function of the simulated time. The stabilization of a DSMC calculation is achieved when this profile becomes jagged and included within a band defined by the standard deviation of the number of simulated particles.

\section{Case studies}

Fig. 2(a) shows the rendering of SpaceLiner-7.1, an advanced concept for a suborbital, hypersonic, winged passenger transport, which is currently under investigation at the German Aerospace Center (Deutsches Zentrum für Luft- und Raumfahrt or DLR $[10,11])$. The fuselage length (L) and the wing span of the vehicle are $63 \mathrm{~m}$ and $40 \mathrm{~m}$, respectively. By referring to a Cartesian system, whose origin is located in the nose stagnation point, the $x$-axis is along the fuselage and the $z$-axis is in the symmetry plane, the center of gravity is located at $x_{\mathrm{cg}}=34.5 \mathrm{~m}$ and $z_{\mathrm{cg}}=1.28 \mathrm{~m}$. Fig. 2(b) shows the vehicle with flaps deflection of $\delta=-35 \mathrm{deg}$, approximated by an unstructured body grid. The surface mesh was generated by the Rhinoceros software, including 1877 and 1961 surface triangles for the clean and flapped configurations, respectively. The wing profile, located at $y=11.5 \mathrm{~m}$ from the symmetry plane, is highlighted in Fig. 2(a) and is shown in Fig. 2(c). The chord (c) and the maximum thickness of the airfoil are about $23.8 \mathrm{~m}$ and $1.1 \mathrm{~m}$, respectively.

The considered flight conditions could likely be a point of interest of a typical mission profile of the SpaceLiner at an altitude of $120 \mathrm{~km}$. The free stream velocity $\left(V_{\infty}\right)$ is $4500 \mathrm{~m} / \mathrm{s}$ [12], the thermodynamic parameters and air composition are provided by the
US standard Atmosphere: $T_{\infty}=329 \mathrm{~K}, \rho_{\infty}=2.24 \times 10^{-8} \mathrm{~kg} / \mathrm{m}^{3}$, $N_{\infty}=5.15 \times 10^{15} \mathrm{~m}^{-3}, p_{\infty}=2.56 \times 10^{-3}[\mathrm{~Pa}], \lambda_{\infty}=3.5 \mathrm{~m}$ and the molar fractions of molecular oxygen, molecular nitrogen and atomic oxygen are $0.0864,0.7294$ and 0.1842 , respectively. The wall temperature $\left(T_{w}\right)$, constant along the whole body, is 300 $\mathrm{K}$. The free stream Mach number is $M_{\infty}=11$, the free stream Reynolds and Knudsen numbers, based on the fuselage length and the airfoil chord, are $\operatorname{Re}_{L \infty}=283, \mathrm{Kn}_{L \infty}=0.06$ and $\operatorname{Re}_{c \infty}=107$, $\mathrm{Kn}_{c \infty}=0.15$; the values of the Knudsen numbers indicate that the flow fields around both the whole vehicle and the airfoil are in low density regime.

Tests at the impact point were carried out in the interval of incidence angle $(\vartheta)$ 5-85 deg with a spacing of 5 deg Each run simulated $10^{5}$ molecule-surface collisions. The tests considered, for both models, specular ( $f=1, \alpha_{n}=\alpha_{t}=0$ ), semi-specular $\left(f=0.5, \alpha_{n}=\alpha_{t}=0.5\right)$ and full diffusive $\left(f=0, \alpha_{n}=\alpha_{t}=1\right)$ interactions.

Simulations on the airfoil were carried out varying the angle of attack AoA in the range $0-50 \mathrm{deg}$ with a spacing of $5 \mathrm{deg}$, considering specular, semi-specular and full diffusive Maxwell models and only the semi-specular CLL model. The computational region for the 2-D simulations, performed by DS2V-64 bits code, was a rectangle: $L_{x 2 D}=29 \mathrm{~m}, L_{z 2 D}=8 \mathrm{~m}$. Using this code, a better geometrical definition of the surface was possible. In fact, the wing section was approximated by 250 linear panels on the upper surface and 250 linear panels on the lower surface.

The simulations on the whole vehicle were carried out in the same interval of angles of attack (0-50 deg) and involved only the specular, semi-specular and full diffusive Maxwell models. The 3-D simulations, performed by the DS3V code, took advantage from the symmetry of the body and of the flow field (no sideslip angle); the flow field was computed about only half vehicle. The computational region was a parallelepiped: $L_{x 3 D}=77 \mathrm{~m}, L_{y 3 D}=$ $28 \mathrm{~m}, L_{z 3 D}=34 \mathrm{~m}$.

\section{Accuracy of the computations}

DS2V and DS3V codes automatically generate the computational grid on the basis of the available megabytes and of free stream number density. The user can control the number of cells 


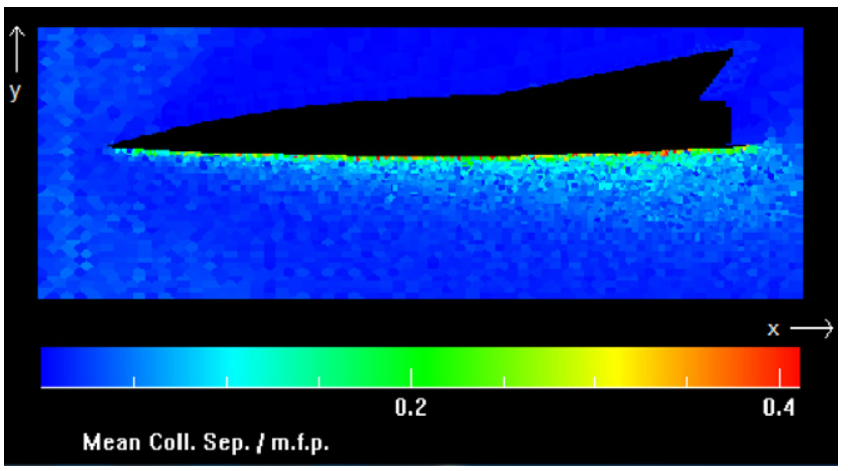

Fig. 3. 2-D spot of the ratio mcs/ $\lambda$ in the meridian plane of the flow field around SpaceLiner with flap: $\alpha=50 \mathrm{deg}$.

Table 1a

Airfoil aerodynamic coefficients in terms of the number of collision cells: $A o A=25$ deg.

\begin{tabular}{llll}
\hline Number of collision cells & $C_{l}$ & $C_{d}$ & $C_{m L E}$ \\
\hline $2.95 \times 10^{6}$ & 0.3475 & 0.6005 & -0.2662 \\
$1.01 \times 10^{6}$ & 0.3423 & 0.5953 & -0.2608 \\
\hline
\end{tabular}

by setting the input number of divisions and elements in each division; the higher is the number of elements and/or the lower is the number of molecule per cell, the higher is the number of the cells after the adaptation process. The codes suggest an optimal number of molecules for adapting both collision and sampling cells.

In the present work the adaptation process for each 2-D test (DS2V) was undertaken considering 30 molecules/cell and about 250 molecules/cell for the collision and sampling cells, respectively; the number of collision and sampling cells were about $9 \times 10^{4}$ and $3 \times 10^{6}$, respectively. The number of simulated particles was about $5 \times 10^{7}$. The quality of the results is guaranteed by the relatively low value (order of $2.0 \times 10^{-3}$ ) of the $\mathrm{mcs} / \lambda$, and by the ratio between simulated time $\left(t_{s}\right)$ and time $\left(t_{\infty}\right)$ to travel the airfoil chord at the free stream velocity $\left(t_{s} / t_{\infty} \cong 5\right)$.

For the three-dimensional computations (DS3V), the adaptation process relied on 8 molecules for each collision cell and on 30 molecules for each sampling cell. Each simulation, with and without flap, was carried out with about $1.6 \times 10^{7}$ simulated particles. The maximum value of the mcs $/ \lambda$ ratio, averaged on all runs, was about 0.6 and $t_{s} / t_{\infty} \cong 50$. For the sake of completeness, Fig. 3 shows the 2-D spots, displayed during the DS3V run, of the ratio $\operatorname{mcs} / \lambda$ at the most severe test condition for DSMC, i.e. for SpaceLiner with flap and at $\alpha=50 \mathrm{deg}$. The maximum value of $\operatorname{mcs} / \lambda$ is less than unity and in most of the flow field close to the suggested optimal limit value of 0.2 .

Even though DS2V and DS3V are well known and widely accepted by the international DSMC community, a set of preliminary runs have been also carried out in order to check the reliability of the grids stated automatically by the codes. A sensitivity analysis has been carried out for both DS2V and DS3V, in terms of the global aerodynamic coefficients computed at an intermediate angle of attack ( $\alpha=25 \mathrm{deg})$. The present analysis follows a similar procedure [25] in which the influence of the number of collision and sampling cells was evaluated on the DS2V results. The test case considered in [25] was the Orion capsule in axi-symmetric flow during re-entry ( $h=85 \mathrm{~km}, V_{\infty}=7600 \mathrm{~m} / \mathrm{s}$ ) and the merit parameter was the heat flux distribution. The present analysis extends the former one by including also DS3V.

Tables $1 \mathrm{a}$ and $1 \mathrm{~b}$ report some aerodynamic coefficients of the airfoil and of the SpaceLiner vehicle computed as functions of the number of collision cells for DS2V and of the input number of elements for DS3V (DS3V does not provide in output the number of
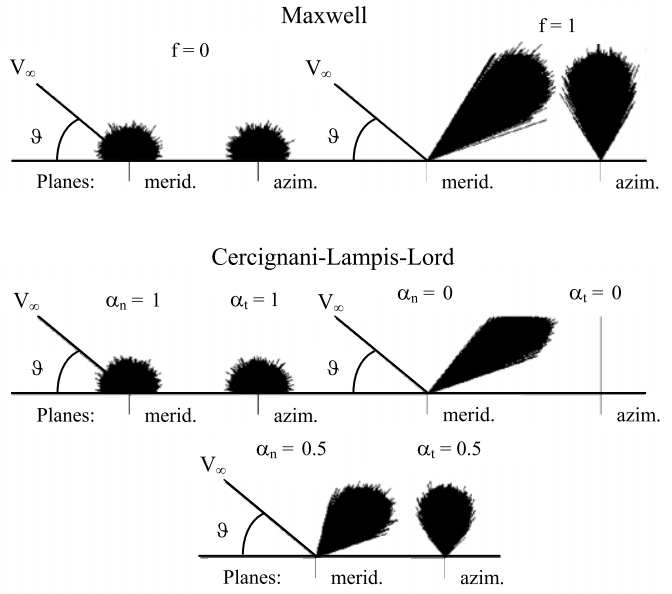

Fig. 4. Re-emission lobes of the Maxwell and the CLL models: $\vartheta=40 \mathrm{deg}$.

Table 1b

SpaceLiner aerodynamic coefficients in terms of the number of elements: AoA $=25$ deg.

\begin{tabular}{llll}
\hline Number of elements & $C_{L}$ & $C_{D}$ & $C_{M c g}$ \\
\hline $2.92 \times 10^{7}$ & 0.7518 & 1.4405 & -0.0831 \\
$5.89 \times 10^{7}$ & 0.7551 & 1.4418 & -0.0825
\end{tabular}

collision and sampling cells). In order to highlight the differences, data are shown up to the fourth decimal digit. Data in the first rows of the tables are computed by the DS2V and DS3V default grid structures; both tables verify that the aerodynamic coefficients of the airfoil and of SpaceLiner practically do not change.

\section{Results and discussion}

\subsection{Impact point}

For a direct comparison of the Maxwell and CLL models, preliminary tests have been carried out considering just the impact point on an elementary surface. Fig. 4 shows the re-emission lobes from the Maxwell and the CLL models at the intermediate angle of incidence of $\vartheta=40 \mathrm{deg}$. The re-emission lobes from both models are drawn in the meridian plane $(x-y)$ and in the azimuth plane $(x-z)$. The graphical representation of the lobes relies on Eqs. (5a)-(5c) for the computation of the incident velocity components and on Eqs. (6a)-(6c) and (9a)-(9c) for the computation of the reemitted velocity components from the Maxwell full diffusive and CLL models, respectively. All re-emitted velocities are scaled in the plots to the free stream velocity.

Figures show that the re-emission lobes corresponding to full diffusive re-emissions are quite similar for the two models and the lobes for specular re-emission in the meridian plane are pretty similar. This is an important result because, being the models completely different, each model can support the other one. The only relevant difference is that the CLL model does not compute re-emission in the azimuth plane for a specular re-emission. For completeness, the typical shape of the lobes computed with the CLL model are shown also for semi-specular $\left(\alpha_{n}=\alpha_{t}=0.5\right)$ re-emission. As expected, also the re-emitted pressure $\left(p_{r}\right)$ and tangential stress $\left(\tau_{r}\right)$ by the two models, scaled by the free stream dynamic pressure, are comparable (see Fig. 5(a) and (b)). The tangential stress are almost equivalent.

To quantify the difference between the models, the ratios between the results of the CLL and Maxwell models are reported in Table 2 . The ratios involving the tangential stresses for full diffusive re-emission are not reported because they are not meaningful. In 

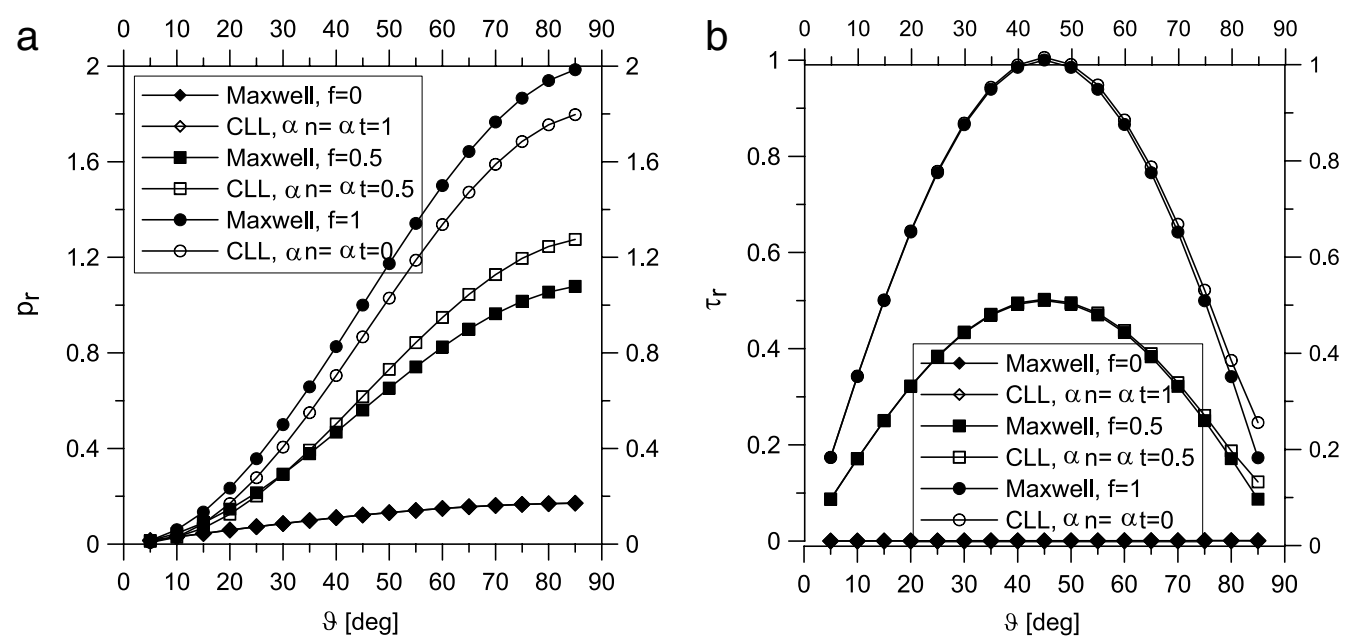

Fig. 5. Profiles of the re-emitted pressure (a) and tangential stress (b) by the Maxwell and CLL models.

Table 2

Ratios of $p_{r}$ and $\tau_{r}$ between the CLL and the Maxwell models.

\begin{tabular}{|c|c|c|c|c|c|}
\hline$\vartheta[\operatorname{deg}]$ & $\begin{array}{l}p_{r C L L} / p_{r M a x w} \\
f=1, \alpha_{n}=\alpha_{t}=0\end{array}$ & $\begin{array}{l}\tau_{r C L L} / \tau_{r M a x w} \\
f=1, \alpha_{n}=\alpha_{t}=0\end{array}$ & $\begin{array}{l}p_{r C L L} / p_{r M a x w} \\
f=\alpha_{n}=\alpha_{t}=0.5\end{array}$ & $\begin{array}{l}\tau_{r C L L} / \tau_{r \operatorname{Maxw}} \\
f=\alpha_{n}=\alpha_{t}=0.5\end{array}$ & $\begin{array}{l}p_{r C L L} / p_{r M a x w} \\
f=0, \alpha_{n}=\alpha_{t}=1\end{array}$ \\
\hline 5 & 0.45 & 1.00 & 0.78 & 1.00 & 1.00 \\
\hline 10 & 0.48 & 1.00 & 0.65 & 1.00 & 1.00 \\
\hline 15 & 0.64 & 1.00 & 0.75 & 1.00 & 1.00 \\
\hline 20 & 0.72 & 1.00 & 0.85 & 1.00 & 1.00 \\
\hline 25 & 0.78 & 1.00 & 0.94 & 1.00 & 1.00 \\
\hline 30 & 0.81 & 1.00 & 1.00 & 1.00 & 1.00 \\
\hline 35 & 0.84 & 1.00 & 1.04 & 1.00 & 1.00 \\
\hline 40 & 0.85 & 1.00 & 1.07 & 1.00 & 1.00 \\
\hline 45 & 0.87 & 1.01 & 1.10 & 1.00 & 1.00 \\
\hline 50 & 0.88 & 1.01 & 1.12 & 1.01 & 1.00 \\
\hline 55 & 0.89 & 1.01 & 1.14 & 1.01 & 1.00 \\
\hline 60 & 0.89 & 1.01 & 1.15 & 1.01 & 1.00 \\
\hline 65 & 0.90 & 1.02 & 1.16 & 1.02 & 1.00 \\
\hline 70 & 0.90 & 1.03 & 1.17 & 1.02 & 1.00 \\
\hline 75 & 0.90 & 1.04 & 1.18 & 1.04 & 1.00 \\
\hline 80 & 0.90 & 1.10 & 1.18 & 1.09 & 1.00 \\
\hline 85 & 0.91 & 1.42 & 1.18 & 1.41 & 1.00 \\
\hline
\end{tabular}

agreement with what shown in Fig. 5 and excluding the low $(5,10$ $\mathrm{deg})$ and the high (80, $85 \mathrm{deg}$ ) angles of incidence, the ratios are all of the order of magnitude one, even for specular re-emission. More specifically, in agreement with the almost coincident full diffusive re-emission lobes, the ratios of pressure are exactly equal to one. The same happens for the ratio of the tangential stresses for specular and semi-specular reflection. This implies that no significant differences are found using the two models. The present results confirm the equivalence of the Maxwell and CLL models already found by Padilla and Boyd [18]. This is an important remark because the computer tests in [18] were performed in rarefied conditions different from the present ones. In fact, Padilla and Boyd carried out computations on flat plate at test conditions corresponding to $140 \mathrm{~km}$ orbit around Venus.

\subsection{Wing section}

Figs. 6(a)-(d) show the influence of the gas-surface interaction model on the wing section aerodynamic coefficients: lift $C_{l}(a)$, drag $C_{d}$ (b), longitudinal moment (the reduction pole is the leading edge) $C_{m}(\mathrm{~d})$, aerodynamic efficiency $\mathrm{E}\left(C_{l} / C_{d}\right)(\mathrm{d})$ in clean configuration ( $\delta=0 \mathrm{deg}$ ). These figures show that, also in this case, the semi-specular Maxwell and CLL models produce very similar results and, as expected, the coefficients for both models are intermediate between the full diffusive and specular cases. This is confirmed also by Fig. 7(a)-(c), showing the profiles of pressure (a), skin friction (b) and heat flux (c) along the wind side of the wing at an intermediate angle of attack AoA $=20 \mathrm{deg}$. The influence, on the computed pressure, of the interaction model is negligible and, as expected, the skin friction and the heat flux for both models is one half of the corresponding value from the full diffusive model.

Tables 3-5 summarize the results in terms of computed coefficients from the Maxwell specular, Maxwell semi-specular and CLL semi-specular model divided by the same coefficients from the Maxwell full diffusive model. According to the previous figures, the effect of the re-emission model is relevant and the ratios increase with the angle of attack. For example, in the interval of angle of attack 0-50 deg, $C_{l f=1} / C_{l f=0}$ changes from 0.77 to $1.68, C_{d f=1} / C_{d f=0}$ changes from 0.07 to 0.98 and $C_{m f=1} / C_{m f=0}$ changes from 0.45 to 1.10. This is explained by the fact that, as shown in Fig. 5(a), increasing the angle of incidence, the specular re-emitted pressure increases much more than the full diffusive one and, consequently, all aerodynamic coefficients (lift, drag, moment) are larger. Furthermore, the specular, re-emitted tangential stress is different from zero, while the full diffusive one is always zero, see Fig. 5(b).

The ratios for the semi-specular Maxwell model show a similar trend but to a lesser extent. Due to the above seen equivalence of the Maxwell and the CLL models, similar values of the ratios are also found for the semi-specular CLL model.

\subsection{SpaceLiner}

Figs. 8(a)-(d) show the profiles of the lift $C_{L}$ (a) and of the drag $C_{D}$ (b) coefficients, of the aerodynamic efficiency $E(c)$, of the 
a
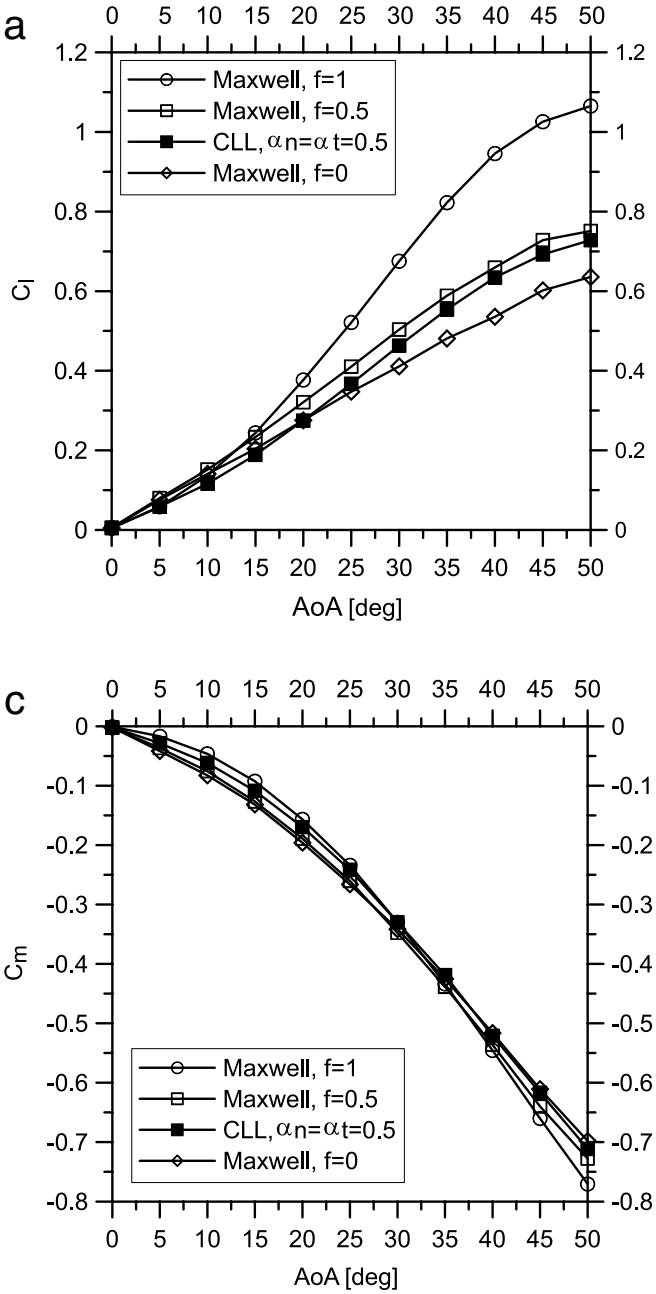
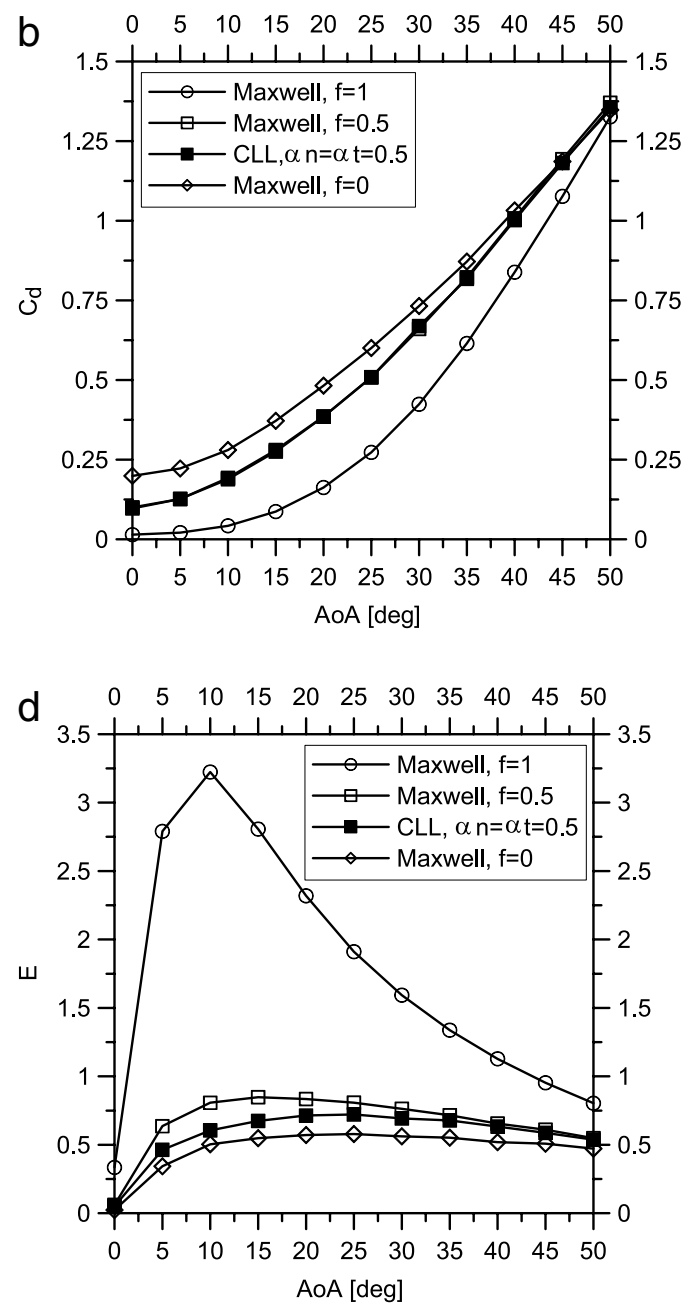

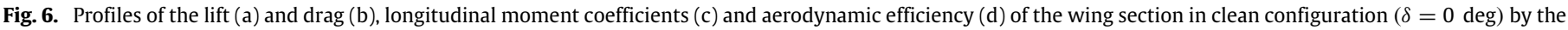
Maxwell and CLL models.

Table 3

Ratios of the airfoil aerodynamic coefficients between the Maxwell specular $(f=1)$ and the full diffusive $(f=0)$ models: $\delta=0 \mathrm{deg}$.

\begin{tabular}{cllc}
\hline AoA [deg] & $C_{l f=1} / C_{l f=0}$ & $C_{m f=1} / C_{m f=0}$ & $E_{f=1} / E_{f=0}$ \\
\hline 0 & 1.07 & 0.45 & 14.59 \\
5 & 0.77 & 0.41 & 8.15 \\
10 & 0.97 & 0.56 & 6.40 \\
15 & 1.20 & 0.70 & 5.12 \\
20 & 1.37 & 0.80 & 4.06 \\
25 & 1.50 & 0.88 & 3.30 \\
30 & 1.64 & 0.97 & 2.84 \\
35 & 1.71 & 1.02 & 2.42 \\
40 & 1.77 & 1.06 & 2.17 \\
45 & 1.70 & 1.08 & 1.88 \\
50 & 1.68 & 1.10 & 1.70
\end{tabular}

longitudinal moment coefficient $C_{M c g}(\mathrm{~d})$, the reduction pole is the vehicle center of gravity, as functions of the angle of attack. Fig. 8(e) shows the stability index I, defined as $I=\left(x_{c g}-x_{p}\right) / L$, where $x_{p}$ is the $x$-coordinate of the pressure center. All results refer to the vehicle without flap deflection and with flap deflection $\delta=$ $-35 \mathrm{deg}$, for different angles of attack.

The increments of the lift and drag coefficients, produced by the flap deflection, are stronger for the specular model, as discussed in the case of the wing section. The most relevant differences, produced by the gas-surface interaction model, are evident in the aerodynamic efficiency and in the longitudinal moment coefficient profile, therefore in the longitudinal stability. It is well known that
Table 4

Ratios of the airfoil aerodynamic coefficients between the Maxwell semi-specular $(f=0.5)$ and the Maxwell full diffusive $(f=0)$ models: $\delta=0$ deg.

\begin{tabular}{cllll}
\hline AoA [deg] & $C_{l f=0.5} / C_{l f=0}$ & $C_{d f=0.5} / C_{d f=0}$ & $C_{m f=0.5} / C_{m f=0}$ & $E_{f=0.5} / E_{f=0}$ \\
\hline 0 & 1.33 & 0.50 & 1.09 & 2.65 \\
5 & 1.06 & 0.57 & 0.88 & 1.86 \\
10 & 1.08 & 0.67 & 0.92 & 1.60 \\
15 & 1.15 & 0.74 & 0.96 & 1.55 \\
20 & 1.17 & 0.80 & 0.96 & 1.46 \\
25 & 1.18 & 0.85 & 0.98 & 1.40 \\
30 & 1.23 & 0.90 & 1.02 & 1.36 \\
35 & 1.22 & 0.94 & 1.03 & 1.30 \\
40 & 1.23 & 0.98 & 1.04 & 1.26 \\
45 & 1.21 & 1.01 & 1.05 & 1.20 \\
50 & 1.18 & 1.02 & 1.04 & 1.16
\end{tabular}

the longitudinal stability is defined by the conditions $d C_{M c g} / d \alpha<$ 0 and $I<0$. SpaceLiner with no flap and with a specular interaction is unstable $\left(d C_{M c g} / d \alpha>0, I>0\right)$ up to $\alpha=40$ deg. On the other hand the vehicle is longitudinally stable in the whole interval of angle of attack for full diffusive and semi-specular interaction models. Considering the results with flaps, even in the condition of longitudinal stability, the equilibrium is more or less stable according to the interaction model.

The interaction model strongly influences the angle of maximum efficiency. This angle, for specular, semi-specular and diffusive models is 5, 20 and 25 deg with no flap deflection and 10, 15 and 20 deg with deflected flap, respectively. As discussed before, 

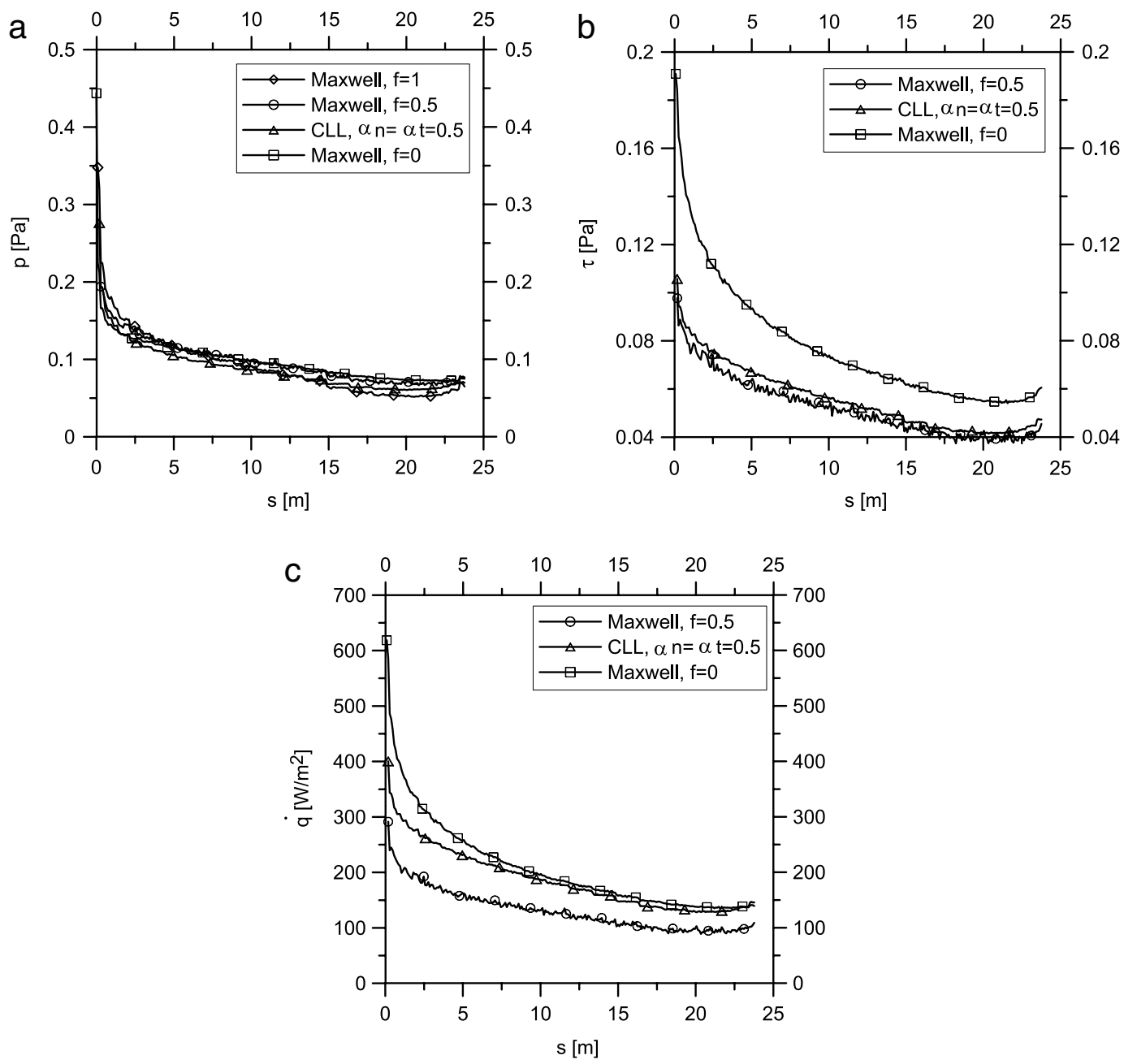

Fig. 7. Profiles of pressure (a), shear stress (b) and heat flux (c) along the wind side of the airfoil by the Maxwell and CLL models: $\alpha=20$ deg, $\delta=0$ deg.

Table 5

Ratios of the airfoil aerodynamic coefficients between the CLL semi-specular $\left(\alpha_{n}=\alpha_{t}=0.5\right)$ and the Maxwell full diffusive $(f=0)$ models: $\delta=0$ deg.

\begin{tabular}{|c|c|c|c|c|}
\hline AoA [deg] & $C_{l \alpha n=\alpha t=0.5} / C_{l f=0}$ & $C_{d \alpha n=\alpha t=0.5} / C_{d f=0}$ & $C_{m \alpha n=\alpha t=0.5} / C_{m f=0}$ & $E_{\alpha n=\alpha t=0.5} / E_{f=0}$ \\
\hline 0 & 1.07 & 0.49 & 0.86 & 2.21 \\
\hline 5 & 0.77 & 0.57 & 0.68 & 1.35 \\
\hline 10 & 0.83 & 0.69 & 0.75 & 1.20 \\
\hline 15 & 0.93 & 0.75 & 0.82 & 1.23 \\
\hline 20 & 1.00 & 0.80 & 0.87 & 1.25 \\
\hline 25 & 1.06 & 0.85 & 0.91 & 1.25 \\
\hline 30 & 1.13 & 0.91 & 0.97 & 1.23 \\
\hline 35 & 1.15 & 0.94 & 0.99 & 1.23 \\
\hline 40 & 1.18 & 0.97 & 1.01 & 1.22 \\
\hline 45 & 1.15 & 1.00 & 1.01 & 1.15 \\
\hline 50 & 1.15 & 1.01 & 1.02 & 1.14 \\
\hline
\end{tabular}

the value of the maximum efficiency angle of attack is important because at this angle a longer downrange and a larger maneuverability is possible along the atmospheric re-entry flight path.

Once again, in order to quantify the influence of the surface interaction model, Tables $6 \mathrm{a}, 6 \mathrm{~b}, 7 \mathrm{a}$ and $7 \mathrm{~b}$ report the ratios of the aerodynamic coefficients and stability index as functions of the angle of attack, computed by the specular and semi-specular models with respect to the full diffusive model. Tables 6a and 7a show the results corresponding to the configuration with no flap deflection. Tables $6 \mathrm{~b}$ and $7 \mathrm{~b}$ refer to the case of deflected flap. These results show that the interaction model has a large effect. For example, the ratio $C_{M c g f=1} / C_{M c g f=0}$ ranges from -0.80 to 0.38 when flap deflection is zero and from 3.29 to 0.94 when the flap is deflected. The ratio $I_{f=1} / I_{f=0}$ is between -4.54 and 11.10 when flap deflection is zero and between -0.16 and 3.30 with deflected flap.

\section{Conclusions and future developments}

The gas-surface interaction models by Maxwell and Cercignani-Lampis-Lord have been compared in hypersonic, rarefied flow, considering the aerodynamic characteristics of the SpaceLiner vehicle. Preliminary comparisons have been carried out analyzing the normal and tangential stresses linked to the re-emitted molecules at the impact point on an elementary surface. The computer simulations have been carried out considering specular, 

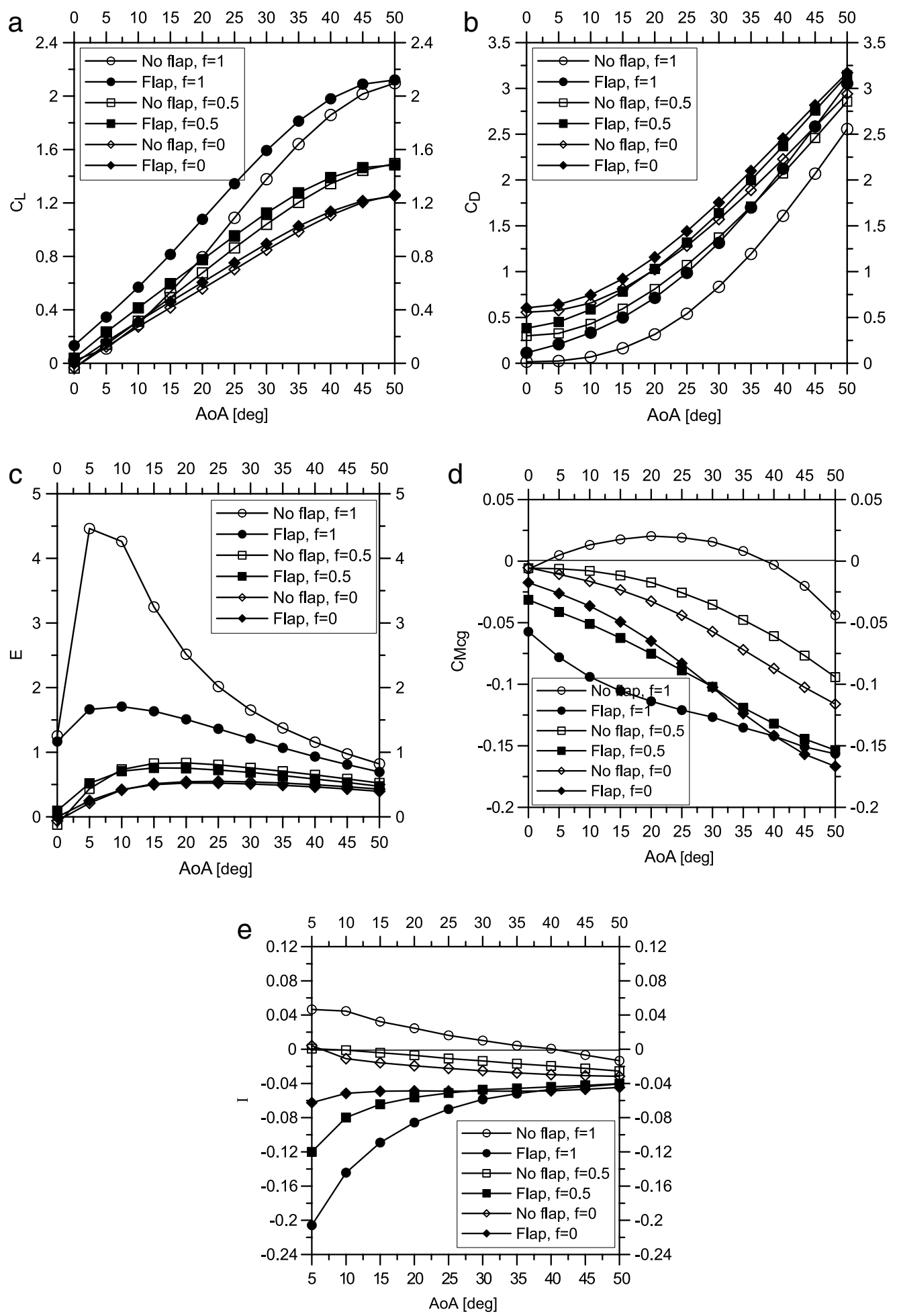

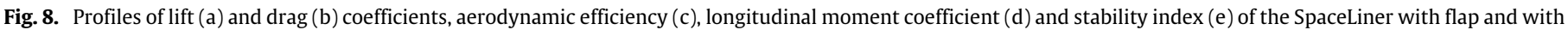
no flap as functions of the angle of attack and the Maxwell models.

semi-specular and full diffusive models. The results show that: (i) the outputs of the Maxwell and Cercignani-Lampis-Lord models are very similar, when the same molecules re-emission behavior is considered, in agreement with what already found in literature, (ii) the influence of the re-emission model (changing from specular to full diffusive) is relevant. The effects increase with the angle of attack and are more important when a complete flapped vehicle configuration is considered. More specifically, considering different interaction models, the angle of maximum efficiency is very different and the longitudinal stability behavior can completely change.

The present results suggest considering in detail the influence of the accommodation coefficients, supposed constant in this preliminary work. Future developments are necessary to investigate the dependence of the accommodation coefficients on the incidence angle. 
Table 6a

Ratios of the SpaceLiner aerodynamic coefficients and stability index by the Maxwell specular $(f=1)$ and the Maxwell full diffusive $(f=0)$ models: $\delta=0$ deg.

\begin{tabular}{|c|c|c|c|c|c|}
\hline AoA [deg] & $C_{L f=1} / C_{L f=0}$ & $C_{D f=1} / C_{D f=0}$ & $C_{M c g f=1} / C_{M c g f=0}$ & $E_{f=1} / E_{f=0}$ & $I_{f=1} / I_{f=0}$ \\
\hline 0 & -0.60 & 0.03 & 1.23 & -20.28 & -4.54 \\
\hline 5 & 0.89 & 0.04 & -0.46 & 20.88 & 11.10 \\
\hline 10 & 1.07 & 0.10 & -0.80 & 10.27 & -4.06 \\
\hline 15 & 1.28 & 0.20 & -0.76 & 6.34 & -2.05 \\
\hline 20 & 1.42 & 0.31 & -0.63 & 4.62 & -1.27 \\
\hline 25 & 1.55 & 0.42 & -0.43 & 3.68 & -0.73 \\
\hline 30 & 1.63 & 0.53 & -0.27 & 3.06 & -0.41 \\
\hline 35 & 1.66 & 0.63 & -0.11 & 2.63 & -0.16 \\
\hline 40 & 1.68 & 0.72 & 0.03 & 2.32 & -0.02 \\
\hline 45 & 1.67 & 0.80 & 0.20 & 2.09 & 0.22 \\
\hline 50 & 1.66 & 0.87 & 0.38 & 1.91 & 0.42 \\
\hline
\end{tabular}

Table 6b

Ratios of the SpaceLiner aerodynamic coefficients and stability index by the Maxwell specular $(f=1)$ and the Maxwell full diffusive $(f=0)$ models: $\delta=-35$ deg.

\begin{tabular}{|c|c|c|c|c|c|}
\hline AoA [deg] & $C_{L f=1} / C_{L f=0}$ & $C_{D f=1} / C_{D f=0}$ & $C_{M c g f=1} / C_{M c g f=0}$ & $E_{f=1} / E_{f=0}$ & $I_{f=1} / I_{f=0}$ \\
\hline 0 & -69.89 & 0.19 & 3.29 & -364.28 & -0.16 \\
\hline 5 & 2.15 & 0.32 & 2.98 & 6.65 & 3.30 \\
\hline 10 & 1.81 & 0.45 & 2.58 & 4.05 & 2.80 \\
\hline 15 & 1.77 & 0.54 & 2.14 & 3.27 & 2.22 \\
\hline 20 & 1.78 & 0.62 & 1.75 & 2.89 & 1.76 \\
\hline 25 & 1.79 & 0.68 & 1.46 & 2.61 & 1.43 \\
\hline 30 & 1.78 & 0.75 & 1.24 & 2.38 & 1.20 \\
\hline 35 & 1.76 & 0.81 & 1.09 & 2.18 & 1.06 \\
\hline 40 & 1.74 & 0.86 & 1.00 & 2.01 & 0.97 \\
\hline 45 & 1.72 & 0.92 & 0.96 & 1.87 & 0.93 \\
\hline 50 & 1.69 & 0.96 & 0.94 & 1.76 & 0.91 \\
\hline
\end{tabular}

Table 7a

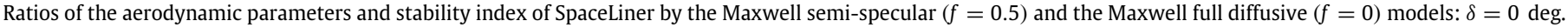

\begin{tabular}{|c|c|c|c|c|c|}
\hline AoA [deg] & $C_{L f=0.5} / C_{L f=0}$ & $C_{D f=0.5} / C_{D f=0}$ & $C_{M c g f=0.5} / C_{M c g f=0}$ & $E_{f=0.5} / E_{f=0}$ & $I_{f=0.5} / I_{f=0}$ \\
\hline 0 & 1.04 & 0.54 & 1.08 & 1.95 & 0.06 \\
\hline 5 & 1.15 & 0.57 & 0.58 & 2.03 & 0.17 \\
\hline 10 & 1.15 & 0.65 & 0.48 & 1.77 & 0.09 \\
\hline 15 & 1.18 & 0.73 & 0.50 & 1.61 & 0.26 \\
\hline 20 & 1.22 & 0.79 & 0.53 & 1.53 & 0.36 \\
\hline 25 & 1.23 & 0.84 & 0.58 & 1.47 & 0.48 \\
\hline 30 & 1.23 & 0.87 & 0.62 & 1.40 & 0.54 \\
\hline 35 & 1.22 & 0.90 & 0.66 & 1.35 & 0.61 \\
\hline 40 & 1.21 & 0.93 & 0.70 & 1.30 & 0.66 \\
\hline 45 & 1.20 & 0.95 & 0.75 & 1.26 & 0.72 \\
\hline 50 & 1.18 & 0.97 & 0.81 & 1.22 & 0.80 \\
\hline
\end{tabular}

Table 7b

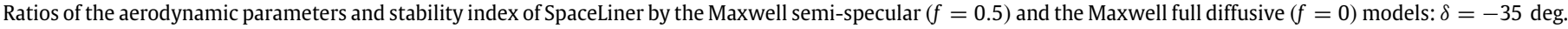

\begin{tabular}{|c|c|c|c|c|c|}
\hline $\mathrm{AOA}[\mathrm{deg}]$ & $C_{L f=0.5} / C_{L f=0}$ & $C_{D f=0.5} / C_{D f=0}$ & $C_{M c g f=0.5} / C_{M c g f=0}$ & $E_{f=0.5} / E_{f=0}$ & $I_{f=0.5} / I_{f=0}$ \\
\hline 0 & -19.21 & 0.63 & 1.80 & -29.81 & -0.25 \\
\hline 5 & 1.45 & 0.70 & 1.58 & 2.07 & 1.92 \\
\hline 10 & 1.32 & 0.79 & 1.40 & 1.68 & 1.55 \\
\hline 15 & 1.29 & 0.85 & 1.27 & 1.52 & 1.31 \\
\hline 20 & 1.28 & 0.89 & 1.16 & 1.44 & 1.15 \\
\hline 25 & 1.27 & 0.91 & 1.07 & 1.39 & 1.04 \\
\hline 30 & 1.26 & 0.93 & 1.00 & 1.35 & 0.97 \\
\hline 35 & 1.24 & 0.95 & 0.96 & 1.30 & 0.93 \\
\hline 40 & 1.22 & 0.97 & 0.93 & 1.27 & 0.90 \\
\hline 45 & 1.20 & 0.98 & 0.92 & 1.23 & 0.90 \\
\hline 50 & 1.19 & 0.99 & 0.92 & 1.20 & 0.90 \\
\hline
\end{tabular}

\section{References}

[1] G.A. Bird, Molecular Gas Dynamics and Direct Simulation Monte Carlo, Clarendon Press, Oxford, Great Britain, 1998.

[2] G.A. Bird, The DSMC Method, Version 1.1, Amazon, Charleston, USA, 2013, ISBS 9781492112907

[3] C. Shen, Rarefied Gas Dynamic: Fundamentals, Simulations and Micro Flows, Springer-Verlag, Berlin, Germany, 2005.

[4] R. Schamberg, A New Analytical Representation of Surface Interaction for Hyper-thermal Free Molecule Flow, Rand Corp, RM-2313, Santa Monica, CA,

[5] R. Schamberg, Analytic Representation of Surface Interaction for Free Molecular Flow with Application to Drag of Various Bodies, Aerodynamics of the Upper Atmosphere, Rand Corp, R-339, Santa Monica, CA, 1959, pp. 12-1-12-41.
[6] S. Nocilla, The surface re-emission law in free molecule flow, in: Proceedings of the 3rd International Symposium on Rarefied Gas Dynamics, Paris, 1960

[7] R.G. Lord, Some extensions to the Cercignani-Lampis gas-surface. scattering kernel, Phys. Fluids A 3 (4) (1991) 706-710.

[8] E.D. Knechtel, W.C. Pitts, Normal and tangential momentum accommodation for Earth satellite conditions, Acta Astronaut. 18 (3) (1973) 171-184.

[9] G.Zuppardi, DSMC implementation of the schamberg model: comparison with the Maxwell model, in: Proceedings of the 24th International Symposium on Rarefied Gas Dynamics, Monopoli, Italy (2004).

[10] M. Sippel, A. van Foreest, C. Bauer, F. Cremaschi, System investigation of the SpaceLiner concept in FAST20XX, AIAA Paper 2011-2294 (2011).

[11] M. Sippel, et al. Technical Maturation of the SpaceLiner Concept, in: 18th AIAA/3AF International Space Planes and Hypersonic Systems and Technologies Conference, 14-18 September 2012, Tours, France, http://dx.doi. org/10.2514/6.2012-5850. 
[12] G. Zuppardi, L. Morsa, M. Sippel, T. Schwanekamp, Aero-thermo-dynamic analysis of the SpaceLiner-7.1 vehicle in high altitude flight, in: Proceedings of the 29th International Symposium on Rarefied Gas Dynamics, Xi'an, China, 2014.

[13] G.A. Bird, The DS2V Program User's Guide Ver. 3.2, G.A.B. Consulting Pty Ltd., Sydney, Australia, 2005.

[14] G.A. Bird, The DS3V Program User's Guide Ver. 2.5, G.A.B. Consulting Pty Ltd., Sydney, Australia, 2006

[15] D.M. Pilinski, Dynamic Gas-Surface Interaction Modeling for Satellite Aerodynamic Computations (Diss. Ph. D. thesis), Univ. of Colorado at Boulder, Boulder CO, 2011

[16] M.M. Moe, S.D. Wallace, K. Moe, Refinements in the determination of satellite drag coefficients: method for resolving density discrepancies. URL http://citeseer.ist.psu.edu/38570.html.

[17] K. Moe, M.M. Moe, Gas-surface interactions in low-earth orbit, Planet. Space Sci. 53 (2005) 793-801.

[18] J.F. Padilla, I.D. Boyd, Assessment of gas-surface interaction models for computation of rarefied hypersonic flow, J. Thermophys. Heat Transf. 23 (2009) 96-105.
[19] M.S. Woronowicz, D.F.G. Rault, Cercignani-Lampis-Lord gas-surface interaction model: Comparison between theory and simulation, J. Spacecraft Rockets 31 (1994) 532-534

[20] R.N. Gupta, J.M. Yos, R.A. Thompson, A review of reaction rates and thermodynamic transport properties for an 11-species air model for chemical and thermal non-equilibrium calculations to 30,000 K, NASA TM 101528, 1989.

[21] G.A. Bird, Sophisticated versus simple DSMC, in: Proceedings of the 25th International Symposium on Rarefied Gas Dynamics, M. Ivanov, A. Rebrov (Eds.), Saint Petersburg, Russia, 2006.

[22] G.A. Bird, M.A. Gallis, J.R. Torczynski, D.J. Rader, Phys Fluid. 21 (2009) 017103

[23] M.A. Gallis, J.R. Torczynski, D.J. Rader, G.A. Bird, J. Comput. Phys. 228 (2009) $4532-4548$

[24] G.A. Bird, The DS2V/3V program suite for DSMC calculations, in: Proceedings of the 24th International Symposium on Rarafied Gas Dynamics, M. Capitelli (Ed.), Monopoli, Italy, 2004.

[25] L. Morsa, G. Zuppardi, R. Votta, A. Schettino, Influence of chemical models on heat flux for EXPERT and orion capsules, J. Aerospace Eng. 228 (2014) 6. 